\title{
Anti-inflammatory Effect of Ginsenoside Rb1 Contributes to the Recovery of Gastrointestinal Motility in the Rat Model of Postoperative Ileus
}

Shanjun Tan, ${ }^{a}$ Wenkui Yu, ${ }^{* a}$ Zhiliang Lin,${ }^{a}$ Qiyi Chen,${ }^{a}$ Jialiang Shi, ${ }^{a}$ Yi Dong, ${ }^{a}$ Kaipeng Duan, ${ }^{a}$ Xiaowu Bai, ${ }^{b}$ Lin $\mathrm{Xu},{ }^{b}$ Jieshou $\mathrm{Li}^{,}{ }^{a}$ and Ning $\mathrm{Li}^{*}, a$

${ }^{a}$ Research Institute of General Surgery, Jinling Hospital, Medical School of Nanjing University; Nanjing 210002, China: and ${ }^{b}$ Research Institute of General Surgery, Jinling Hospital, Clinical School of Nanjing, Second Military Medical University; Nanjing 210002, China.

Received June 12, 2014; accepted August 25, 2014; advance publication released online August 30, 2014

Ginsenoside Rb1 (GRb1), one of the principle active components of Panax ginseng, has been reported to reduce inflammation in various diseases. In the present study, we investigated whether GRb1 has an antiinflammatory effect on postoperative ileus (POI) and further contributes to the recovery of gastrointestinal motility. POI was induced in rats by intestinal manipulation. The POI rats received 5, 10 and $20 \mathrm{mg} / \mathrm{kg} \mathrm{GRb1}$ orally via gavage four times before and after surgery. Gastrointestinal motility was assessed by charcoal transport. Systemic inflammation was assessed by serum tumor necrosis factor (TNF)- $\alpha$, interleukin (IL)-1 $\beta$, IL-6 and IL-10 concentrations, whereas intestinal inflammation was assessed by the activity of myeloperoxidase, and concentrations and gene expression of TNF- $\alpha$, IL-1 $\beta$, IL-6 and IL-10 in the ileum tissue. The results revealed that GRb1 increased rat gastrointestinal transit with POI. The increased levels of systemic and intestinal inflammatory parameters in POI rats were also reduced by GRb1. In addition, GRb1 reduced systemic and intestinal inflammation and increased the gastrointestinal transit of POI rats in a dose-dependent manner, and with significance at doses of 10 and $20 \mathrm{mg} / \mathrm{kg}$. These results suggest that GRb1 has a potent anti-inflammatory effect on POI and further contributes to the recovery of gastrointestinal motility. GRb1 may be a promising treatment for POI prophylaxis.

Key words ginsenoside Rb1; postoperative ileus; inflammation

Ginseng, the dried root of Panax ginseng C. A. Meyer (Araliaceae), has been well accepted as a tonic to treat many disorders in Chinese traditional medicine. It is considered a famous herb since the earliest Chinese pharmaceutical monograph "Shen Nong Ben Cao Jing."1) Nowdays, ginseng has been worldwide used as a popular traditional herbal medicine, especially in China, Korea and Japan. Ginsenosides are thought to be the main active components of ginseng with multiple pharmacological activities including anti-inflammation, anti-aging, anti-tumor, anti-oxidation, and anti-fatigue. ${ }^{2-8)}$ Modern science has identified more than 50 kinds of ginsenosides. Ginsenoside Rb1 (GRb1), one of the main ginsenosides, belongs to the protopanaxadiol group of steroidal saponins. It is reported that GRb1 has been frequently used to reduce inflammatory process in various diseases. ${ }^{9-13)}$

Currently, one of the major tasks of surgery is to accelerate postoperative rehabilitation. Postoperative ileus (POI) is a common and severe iatrogenic complication after abdominal surgery. ${ }^{14,15)}$ It is characterized by gastrointestinal dysmotility. ${ }^{16)}$ For affected patients, POI manifests itself as some main symptoms including abdominal bloating and distension, a mix of nausea and vomiting and delayed return of flatus and stool passage. ${ }^{17,18)}$ Patients who suffer from POI have a long time to recover from this gastrointestinal motility disorders. POI prolongs patient's hospitalization, increases medical costs, and therefore affects postoperative rehabilitation. ${ }^{19,20)}$ However, the etiology of POI has not been fully explained. ${ }^{16)}$

Much of the published research suggests that many factors, such as neurogenic reflexes, inhibitory agents and inflammation, are responsible for the development and progression of

The authors declare no conflict of interest.
POI. ${ }^{16,21,22)}$ Among these factors, accumulating evidence indicates that intestinal inflammation plays an important role in the pathophysiology of POI. ${ }^{16,23,24)}$ The extent of intestinal manipulation during abdominal surgery causes the degree of intestinal inflammation and further determines the duration and severity of gastrointestinal dysmotility. ${ }^{25)}$ Therefore, methods to reduce intestinal inflammation and shorten postoperative rehabilitation course are popular pursuits in modern surgery. ${ }^{16)}$

In the present study, therefore, we used charcoal transport and some typical inflammatory parameters to investigate whether GRb1 has an anti-inflammatory effect on POI and further contributes to the recovery of gastrointestinal motility.

\section{MATERIALS AND METHODS}

Animals The protocol for the animal experiment was approved by the Institutional Animal Care and Use Committee of Jinling Hospital, Nanjing University. Healthy adult male Sprague-Dawley rats (weighing $220 \pm 10$ g) were obtained from Jinling Hospital in Nanjing, Jiangsu, China. All rats received human care throughout the experiment in accordance with "Guide for the Care and Use of Laboratory Animals." The rats were housed in our laboratory with free access to standard rat chow and tap water made available ad libitum. The lights were maintained on a $12: 12$-h light:dark temperature controlled environment.

Drugs GRb1 (purity over 98\%) was purchased from Shanghai Tauto Biotech Co., Ltd., Shanghai, China. Charcoal and gum arabic were purchased from Shanghai Boyun Biotech Co., Ltd., Shanghai, China. All chemicals and reagents were procured from local suppliers and were of analytical grade.

Animal Grouping and Administration After an adapta- 
tion period for one week, 48 rats were randomly divided into 6 groups: a control group (CG), a POI model group (MG), three GRb1-treated POI model groups at a dose of 5, 10 and $20 \mathrm{mg} / \mathrm{kg}$, respectively (GG, including GG5, GG10 and GG20), and a control group treated with GRb1 at a dose of $20 \mathrm{mg} / \mathrm{kg}$ (CG20), with 8 in each. The rat model of POI was induced by intestinal manipulation as described in the previous study. ${ }^{26,27)}$ Briefly, after full anesthesia with $2 \%$ pentobarbital sodium $(3.5 \mathrm{~mL} / \mathrm{kg})$, rats in the $\mathrm{MG}$ and the GG groups were laparotomized through a midline abdominal incision, and then the small bowel was manipulated with moderate compression between two moist cotton applicators along its entire length. The $\mathrm{CG}$ and the CG20 groups went through the same procedure, but without any intestinal manipulation. Rats in the CG and the MG groups received saline orally via gavage once daily at $3 \mathrm{~d}, 2 \mathrm{~d}$ and $1 \mathrm{~d}$ before surgery, and at $6 \mathrm{~h}$ after surgery. ${ }^{28)}$ Rats in three GG groups were separately administrated GRb1 dissolved in saline at a dose of 5,10 and $20 \mathrm{mg} / \mathrm{kg}$ four times by the same method as the CG group. ${ }^{29)}$ Rats in the CG20 group were also similarly administrated GRbl at a dose of $20 \mathrm{mg} / \mathrm{kg}$ four times by the same method as the CG group.

Determination of Gastrointestinal Motility and Sampling To explore an appropriate investigation time point, we did a pilot experiment prior to this study, demonstrating that the levels of intestinal inflammation and motility differed significantly at $24 \mathrm{~h}$ after surgery, but no significant differences were found on postoperative day 2 or later (data not shown). Therefore, at $24 \mathrm{~h}$ after surgery, all rats in the 6 groups were selected to assess gastrointestinal motility by charcoal transport as described in the previous study. ${ }^{30)}$ Briefly, rats were administered with a black marker $(10 \%$ charcoal suspension in $10 \%$ gum arabic, $10 \mathrm{~mL} / \mathrm{kg}$ body weight) by gavage. Twenty minutes later, rats were fully anesthetized by subcutaneous injection of $2 \%$ pentobarbital sodium $(3.5 \mathrm{~mL} / \mathrm{kg})$. Blood were obtained immediately from the inferior vena cava, and then the small bowel was obtained from pylorus to cecum. The distance travelled by the marker in the small bowel was measured in centimeter and reported as a percentage of total length of the small bowel. Subsequently, a segment of the complete ileum tissue ( $2 \mathrm{~cm}$ from the ileocecal valve) was harvested for further analyses of cytokines, gene expression, and myeloperoxidase (MPO) activity.

Determination of Concentrations of Cytokines in Serum and the Ileum Tissue The serum waspreparedbycentrifugation at a speed of $800 \times \boldsymbol{g}$, at $4^{\circ} \mathrm{C}$ for $15 \mathrm{~min}$. The ileum tissue was homogenized, centrifuged at a speed of $2000 \times \boldsymbol{g}$, at $4^{\circ} \mathrm{C}$ for $15 \mathrm{~min}$, and then the supernatant was obtained. The protein concentration in the supernatant was determined using the method of Bradford. ${ }^{31)}$ The concentrations of tumour necrosis factor (TNF)- $\alpha$, interleukin (IL)-1 $\beta$, IL-6 and IL-10 in serum and the ileum tissue were determined with an ELISA kit for rats (R\&D Systems, Germany) according to the manufacturer's instructions. Values were expressed as $\mathrm{pg} / \mathrm{mL}$ in serum or $\mathrm{pg} / \mathrm{g}$ in the ileum tissue.

Analysis of Expression of Cytokine Gene in the Ileum Tissue The separate total RNA from ileum tissue was extracted using Trizol reagent (Invitrogen, U.S.A.) according to the manufacturer's instructions. Target mRNAs of TNF- $\alpha$, IL-1 $\beta$, IL-6 and IL-10 were separately reverse-transcribed to complementary DNA and measured using real-time polymerase chain reaction (RT-PCR) as described in our previ-
Table 1. The Primer Sequences Used for RT-PCR

\begin{tabular}{clc}
\hline \hline Gene & & \multicolumn{1}{c}{ Primer sequence } \\
\hline TNF- $\alpha$ & Forward & 5'-ACTCCCAGAAAAGCAAGCAA-3' \\
& Reverse & 5'-CGAGCAGGAATGAGAAGAGG-3' \\
IL-1 $\beta$ & Forward & 5'-AGGCTTCCTTGTGCAAGTGT-3' \\
& Reverse & 5'-TGAGTGACACTGCCTTCCTG-3' \\
IL-6 & Forward & 5'-CTGCTCTGGTCTTCTGGAGT-3' \\
& Reverse & 5'- GGTCTTGGTCCTTAGCCACT-3' \\
IL-10 & Forward & 5'-ATAACTGCACCCACTTCCCA-3' \\
& Reverse & 5'- TTTCTGGGCCATGGTTCTCT-3' \\
GAPDH & Forward & 5'-GGCATTGCTCTCAATGACAA-3' \\
& Reverse & 5'-TGTGAGGGAGATGCTCAGTG-3' \\
\hline
\end{tabular}

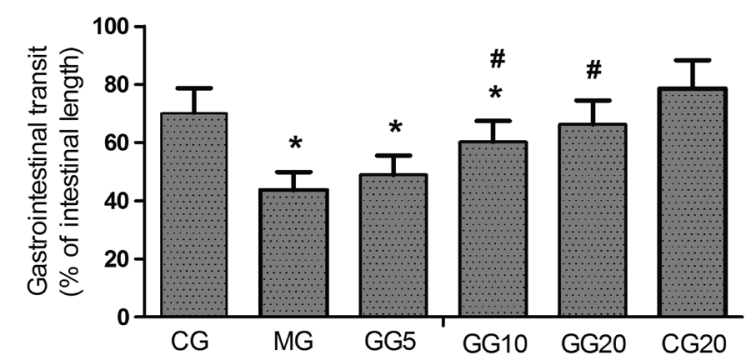

Fig. 1. Effects of GRb1 on Gastrointestinal Motility

CG (control group), MG (POI model group), GG5, GG10 and GG20 (ginsenoside Rbl-treated POI model group at a dose of 5,10 and $20 \mathrm{mg} / \mathrm{kg}$, respectively), CG20 (control group treated with GRb1 at a dose of $20 \mathrm{mg} / \mathrm{kg}$ ). Values are expressed as the mean \pm S.D. $* p<0.05$ means the significance of MG, GG and CG20 compared with $\mathrm{CG}$, and ${ }^{\#} p<0.05$ means the significance of GG compared with MG, respectively.

ous study. ${ }^{23,32)}$ Glyceraldehyde-3-phosphate dehydrogenase (GAPDH) was used as an internal standard to normalize the target mRNAs, and relative quantifications and calculations were performed by the $2^{-\Delta \Delta C T}$ method to analyze gene expression. ${ }^{33)}$ The primer sequences were shown in Table 1.

Assessment of MPO Activity in the Ileum Tissue The ileum tissue was homogenized, centrifuged at a speed of $2000 \times \mathbf{g}$, at $4^{\circ} \mathrm{C}$ for $15 \mathrm{~min}$, and then the supernatants were obtained. The protein concentration in the supernatant was determined using the method of Bradford. ${ }^{31)}$ MPO activity was quantitatively measured by spectrophotometry at $460 \mathrm{~nm}$ as described in our previous study. ${ }^{32)}$ Values were expressed as units/g in the ileum tissue.

Statistical Analysis Data were expressed as mean \pm standard deviation (S.D.). Statistical analyses were performed using the SPSS for Windows (version 16.0) statistical program. After being analyzed by homogeneity test for variance, all the data were analyzed. The significance of the mean difference was determined by one-way ANOVA, followed by the least significant difference test for multigroup comparisons. Differences were considered significant if the $p$ value was lower than 0.05 .

\section{RESULTS}

Effects of GRb1 on Gastrointestinal Motility The results were shown in Fig. 1. There were no significant differences in gastrointestinal transit between the CG and the CG20 groups $(p>0.05)$. The gastrointestinal transit of the MG group was significantly decreased $(p<0.05)$ when compared with that of the CG group. However, GRb1 induced a progressive increase in the gastrointestinal transit in a dose-dependent 

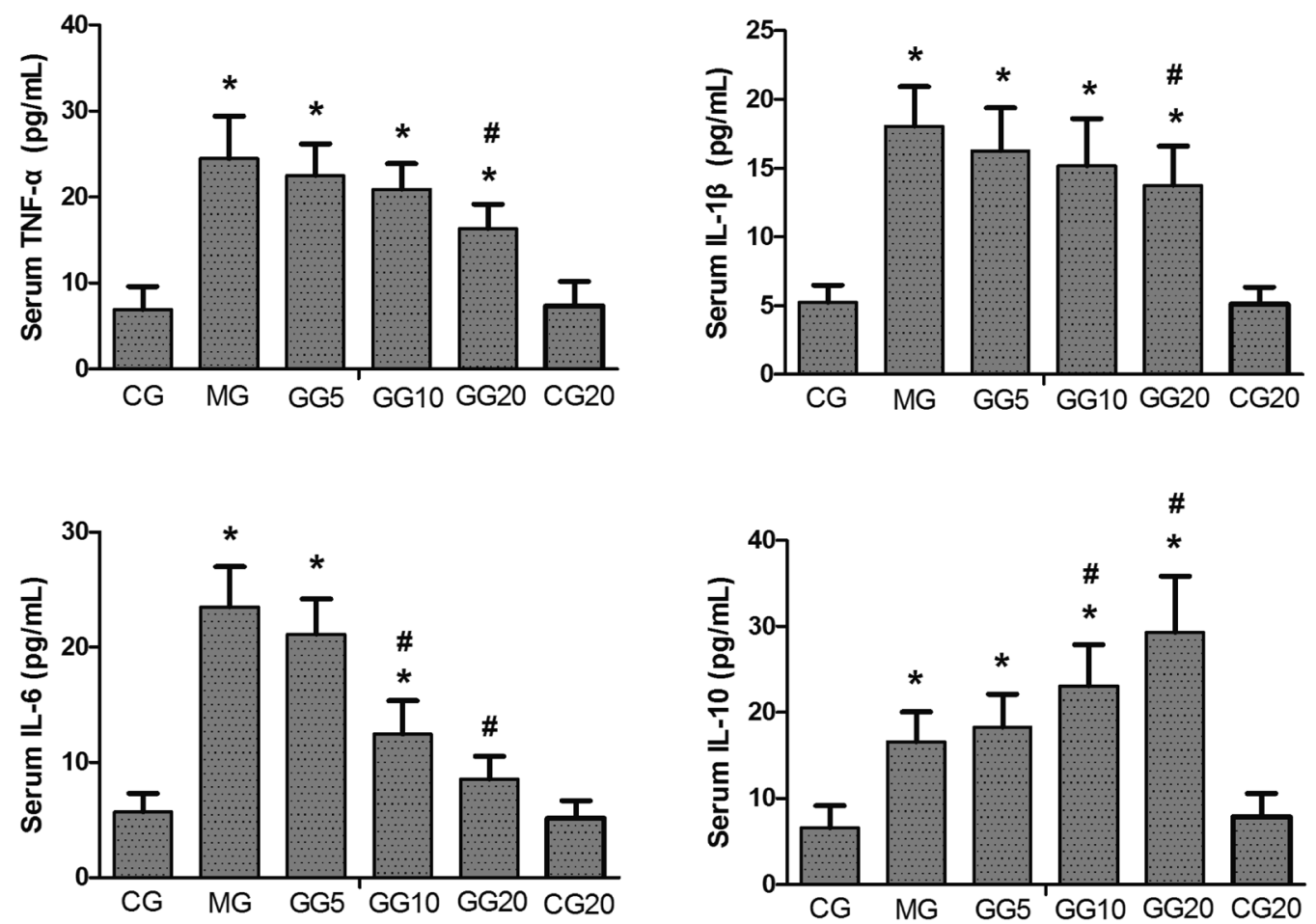

Fig. 2. Effects of GRb1 on Concentrations of TNF- $\alpha$, IL-1 $\beta$, IL-6 and IL-10 in Serum

CG (control group), MG (POI model group), GG5, GG10 and GG20 (ginsenoside Rb1-treated POI model group at a dose of 5, 10 and 20 mg/kg, respectively), CG20 (control group treated with GRb1 at a dose of $20 \mathrm{mg} / \mathrm{kg}$ ). Values are expressed as the mean \pm S.D. * $p<0.05$ means the significance of MG, GG and CG20 compared with $\mathrm{CG}$, and ${ }^{\#} p<0.05$ means the significance of GG compared with $\mathrm{MG}$, respectively.

manner, differed significantly in the GG10 and the GG20 groups when compared with that in the MG group $(p<0.05)$. In addition, while the gastrointestinal transit of the GG5 and the GG10 groups was still significantly slower than that of the CG group $(p<0.05)$, that of the GG20 group returned to the level of the $\mathrm{CG}$ group.

Effects of GRb1 on Concentrations of TNF- $\alpha$, IL-1 $\beta$, IL-6 and IL-10 in Serum The results were shown in Fig. 2. There were no significant differences in the serum concentrations of TNF- $\alpha$, IL- $1 \beta$, IL- 6 and IL-10 between the CG and the CG20 groups $(p>0.05)$. The serum concentrations of TNF- $\alpha$, IL- $1 \beta$, IL- 6 and IL-10 of the MG group were significantly increased $(p<0.05)$ when compared with those of the CG group. However, GRb1 induced a progressive decrease in the serum concentrations of pro-inflammatory cytokine TNF$\alpha$, IL- $1 \beta$ and IL-6 in a dose-dependent manner. The serum concentrations of TNF- $\alpha$ and IL- $1 \beta$ of the GG20 group were significantly decreased when compared with those of the MG group $(p<0.05)$, although the serum concentrations of TNF- $\alpha$ and IL- $1 \beta$ in all three GG groups were still significantly higher than those in the CG group $(p<0.05)$. The serum concentration of IL- 6 of the GG10 and the GG20 groups was significantly decreased when compared with that in the $\mathrm{MG}$ group $(p<0.05)$, and the serum concentration of IL- 6 of the GG20 group returned to the level of the CG group, although the serum concentration of IL-6 of the GG5 and the GG10 groups was still significantly higher than that of the CG group $(p<0.05)$. In addition, GRbl also induced a progressive increase in the serum concentration of anti-inflammatory cytokine IL-10 in a dose-dependent manner, differed significantly in the GG10 and the GG20 groups when compared with that in the MG group $(p<0.05)$.
Effects of GRb1 on Concentrations of TNF- $\alpha$, IL-1 $\beta$, IL-6 and IL-10 in the Ileum Tissue The results were shown in Fig. 3. There were no significant differences in the concentrations of TNF- $\alpha$, IL- $1 \beta$, IL- 6 and IL-10 in the ileum tissue between the $\mathrm{CG}$ and the CG20 groups $(p>0.05)$. The tissue concentrations of TNF- $\alpha$, IL- $1 \beta$, IL- 6 and IL-10 of the MG group were significantly increased $(p<0.05)$ when compared with those of the CG group. However, GRb1 induced a progressive decrease in the tissue concentrations of proinflammatory cytokine TNF- $\alpha$, IL- $1 \beta$ and IL-6 in a dosedependent manner. The tissue concentrations of TNF- $\alpha$ and IL- $1 \beta$ of the GG10 and the GG20 groups were significantly decreased when compared with those of the MG group $(p<0.05)$, and the tissue concentrations of TNF- $\alpha$ and IL- $1 \beta$ of the GG20 group returned to the level of the CG group, although the tissue concentrations of TNF- $\alpha$ and IL- $\beta$ in the GG5 and the GG10 groups were still significantly higher than those in the CG group $(p<0.05)$. The tissue concentration of IL- 6 of the GG10 and the GG20 groups was significantly decreased when compared with that in the MG group $(p<0.05)$, and returned to the level of the CG group, although the tissue concentration of IL-6 in the GG5 group was still significantly higher than that in the CG group $(p<0.05)$. In addition, GRb1 also induced a progressive increase in the tissue concentration of anti-inflammatory cytokine IL-10 in a dose-dependent manner, differed significantly in the GG10 and the GG20 groups when compared with that in the MG group $(p<0.05)$.

Effects of GRb1 on Gene Expression of TNF- $\alpha$, IL-1 $\beta$, IL-6 and IL-10 in the Ileum Tissue The results were shown in Fig. 4. There were no significant differences in the gene expression of TNF- $\alpha$, IL- $1 \beta$, IL- 6 and IL-10 in the ileum tissue between the $\mathrm{CG}$ and the CG20 groups $(p>0.05)$. The 

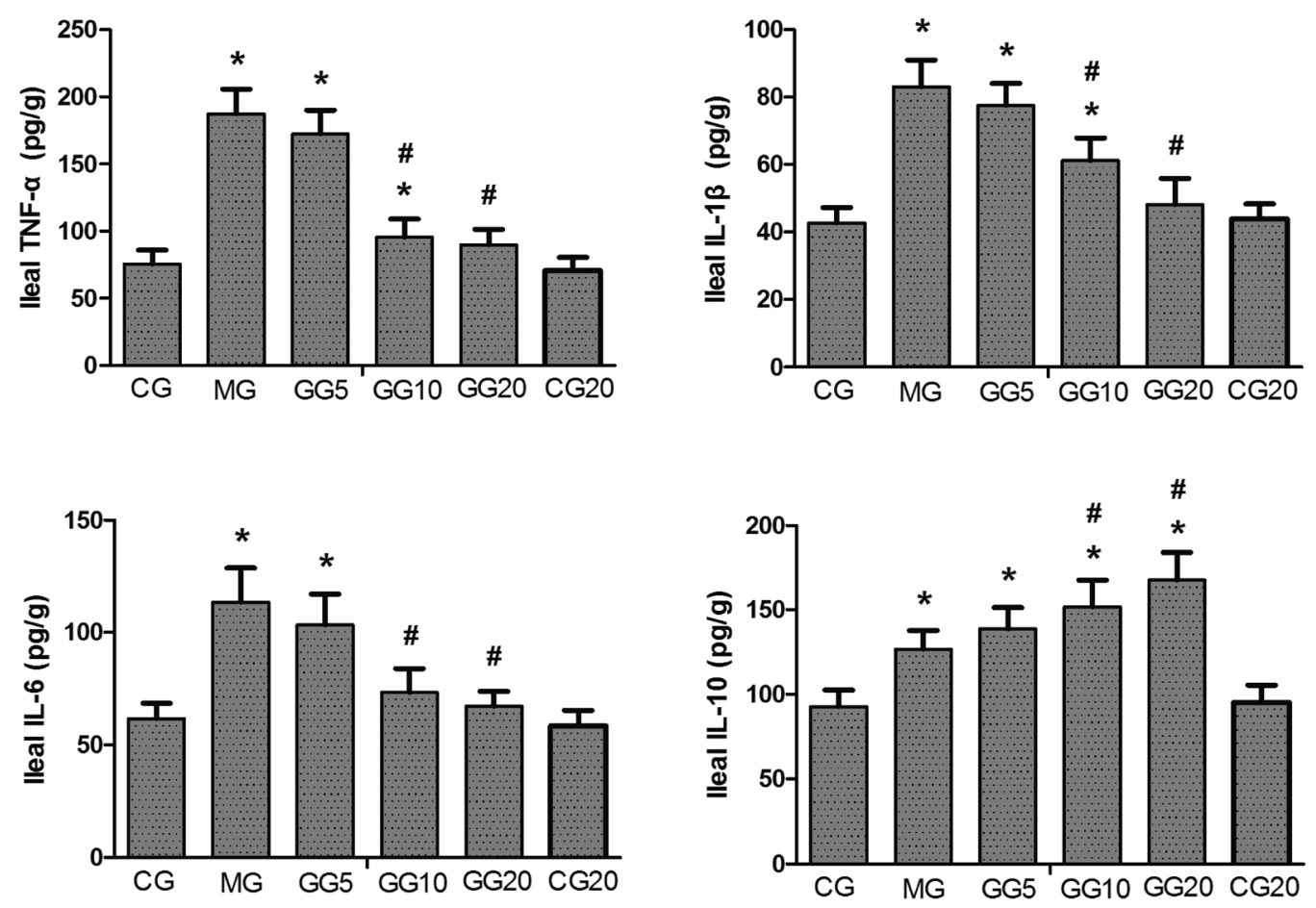

Fig. 3. Effects of GRb1 on Concentrations of TNF- $\alpha$, IL-1 $\beta$, IL-6 and IL-10 in the Ileum Tissue

CG (control group), MG (POI model group), GG5, GG10 and GG20 (ginsenoside Rb1-treated POI model group at a dose of 5, 10 and $20 \mathrm{mg} / \mathrm{kg}$, respectively), CG20 (control group treated with GRb1 at a dose of $20 \mathrm{mg} / \mathrm{kg}$ ). Values are expressed as the mean \pm S.D. * $p<0.05$ means the significance of MG, GG and CG20 compared with $\mathrm{CG}$, and ${ }^{\#} p<0.05$ means the significance of GG compared with $\mathrm{MG}$, respectively.
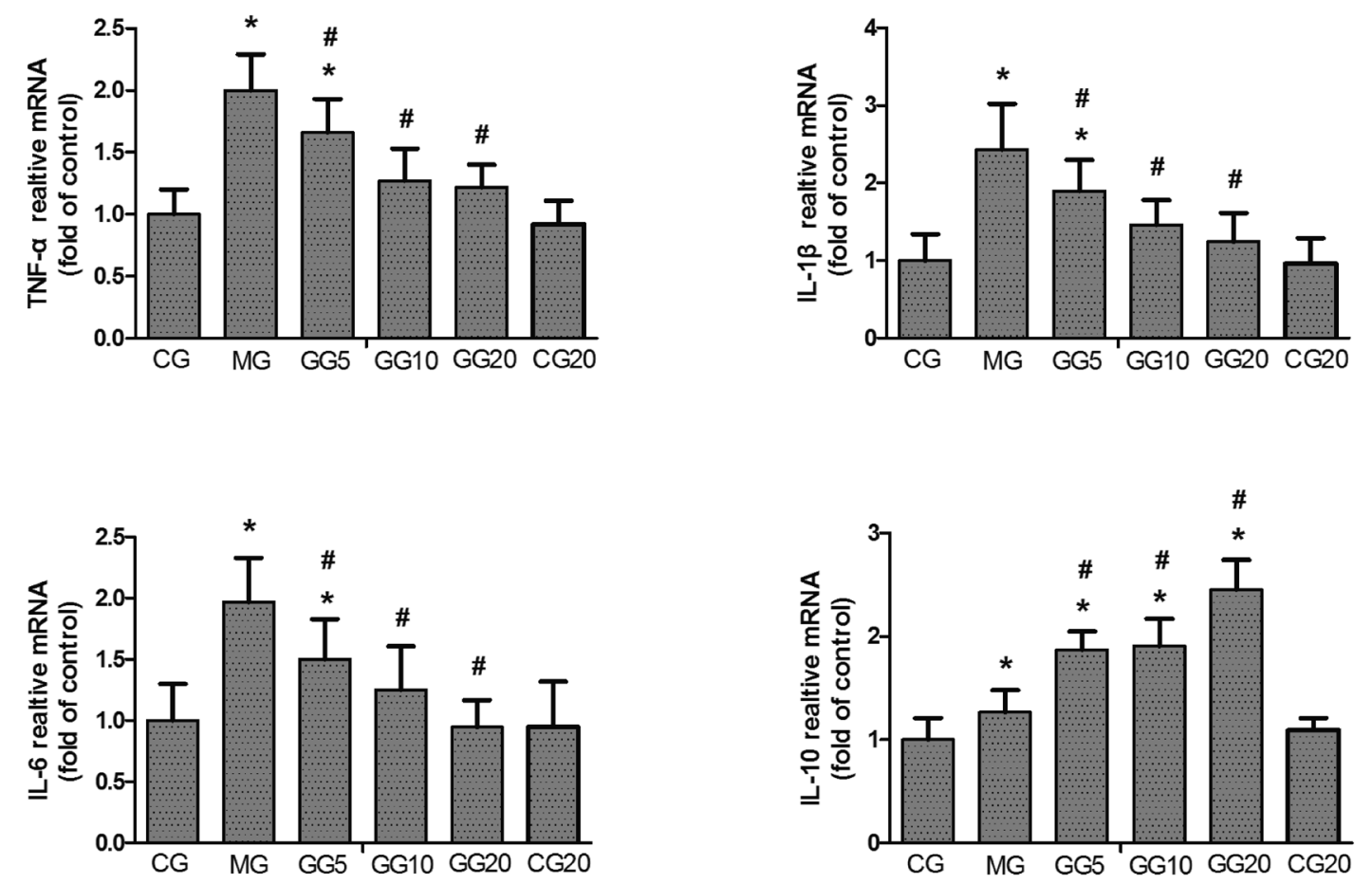

Fig. 4. Effects of GRb1 on Gene Expression of TNF- $\alpha$, IL-1 $\beta$, IL-6 and IL-10 in the Ileum Tissue

CG (control group), MG (POI model group), GG5, GG10 and GG20 (ginsenoside Rb1-treated POI model group at a dose of 5, 10 and $20 \mathrm{mg} / \mathrm{kg}$, respectively), CG20 (control group treated with GRb1 at a dose of $20 \mathrm{mg} / \mathrm{kg}$ ). Values are expressed as the mean \pm S.D. * $p<0.05$ means the significance of MG, GG and CG20 compared with $\mathrm{CG}$, and ${ }^{\#} p<0.05$ means the significance of $\mathrm{GG}$ compared with $\mathrm{MG}$, respectively.

gene expression of TNF- $\alpha$, IL-1 $\beta$, IL- 6 and IL-10 of the MG group was significantly up-regulated $(p<0.05)$ when compared with that of the CG group. However, GRb1 induced a progressive down-regulation in the gene expression of proinflammatory cytokine TNF- $\alpha$, IL-1 $\beta$ and IL-6 in a dose-de- pendent manner, differed significantly in all three GG groups when compared with that in the MG group $(p<0.05)$. In addition, while the gene expression of TNF- $\alpha$, IL- $1 \beta$ and IL- 6 in the GG5 group was still significantly higher than that in the CG group $(p<0.05)$, the gene expression of TNF- $\alpha$, IL- $1 \beta$ and 


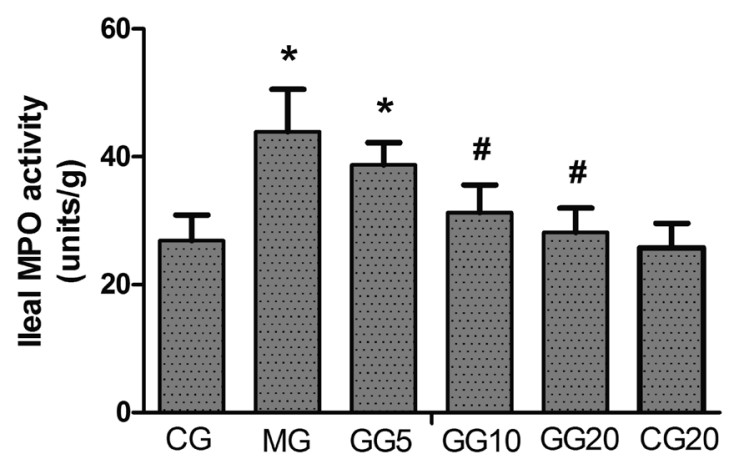

Fig. 5. Effects of GRb1 on MPO Activity in the Ileum Tissue

CG (control group), MG (POI model group), GG5, GG10 and GG20 (ginsenoside Rb1-treated POI model group at a dose of 5,10 and $20 \mathrm{mg} / \mathrm{kg}$, respectively), CG20 (control group treated with GRb1 at a dose of $20 \mathrm{mg} / \mathrm{kg}$ ). Values are expressed as the mean \pm S.D. ${ }^{*} p<0.05$ means the significance of MG, GG and CG20 compared with $\mathrm{CG}$, and ${ }^{\#} p<0.05$ means the significance of GG compared with $\mathrm{MG}$, respectively.

IL-6 of the GG10 and the GG20 groups returned to the level of the CG group. In addition, GRb1 also induced a progressive up-regulation in the gene expression of anti-inflammatory cytokine IL-10 in a dose-dependent manner, differed significantly in three GG groups when compared with that in the MG group $(p<0.05)$.

Effects of GRb1 on MPO Activity in the Ileum Tissue The results were shown in Fig. 5. There were no significant differences in the MPO activity in the ileum tissue between the CG and the CG20 groups $(p>0.05)$. The MPO activity of the MG group was significantly increased $(p<0.05)$ when compared with that of the CG group. However, GRbl induced a progressive decrease in the MPO activity in a dose-dependent manner, differed significantly in the GG10 and the GG20 groups when compared with that in the MG group $(p<0.05)$. In addition, while the MPO activity of the GG5 group was still significantly lower than that of the CG group $(p<0.05)$, that of the GG10 and the GG20 groups returned to the level of the CG group.

\section{DISCUSSION}

In the present study, to investigate whether GRb1 has an anti-inflammatory effect on POI and further contributes to the recovery of gastrointestinal motility, we used charcoal transport to assess gastrointestinal motility and some typical inflammatory parameters were determined. The results showed that GRb1 could increase gastrointestinal transit, reduce the level of systemic and intestinal inflammatory parameters in POI rats in a dose-dependent manner.

POI is commonly followed by abdominal surgery, leading to increased patient morbidity and prolonged rehabilitation. ${ }^{14,15)}$ It is well known that POI is characterized by gastrointestinal dysmotility involved in response to surgical stress. ${ }^{16)}$ Enhancement of gastrointestinal motility will significantly improve POI and enhance recovery after surgery. ${ }^{15,34)}$ Therefore, to investigate the improvement effect of GRbl on POI, it is important to assess gastrointestinal motility. In the previous study, charcoal transport is commonly used as an objective measurement to assess gastrointestinal motility in animal research. ${ }^{23,30,35,36)}$ These studies demonstrate that POI is associated with decreased gastrointestinal transit assessed by charcoal transport. In the present study, we also employed charcoal transport to determine the gastrointestinal motility, and further investigate the improvement effect of GRb1 on POI. The results showed that GRb1 had no direct effect on gastrointestinal transit rate with healthy animals, and therefore the possibility has been excluded that GRb1 can directly stimulate intestinal motility. However, gastrointestinal transit was decreased in POI rats, but GRb1 increased gastrointestinal transit of POI rats, and the effect is dose-dependent. These findings suggested that GRb1 has beneficial effects on the recovery of gastrointestinal motility in POI.

It is well accepted that intestinal inflammation plays a vital role in the development and progression of POI. ${ }^{16)}$ A postoperative increase in intestinal inflammation has an positive correlation with the severity of POI, ${ }^{23,25,26)}$ suggesting that decreased intestinal inflammation may improve POI. Therefore, to further study the mechanism of the contribution of GRb1 to the recovery of gastrointestinal motility, we investigate the anti-inflammatory effect of GRb1 on POI. In the previous study, pro-inflammatory cytokine TNF- $\alpha$, IL- $1 \beta$ and IL- 6 are reported to be rapidly produced in the intestinal inflammatory process during induction of POI in animals. ${ }^{27,37,38)}$ These cytokines act as inhibitors and contribute to the gastrointestinal dysmotility through their direct cytotoxic action or their effect on the production of nitric oxide and prostanoids. ${ }^{24)}$ In contrast to these pro-inflammatory cytokines, anti-inflammatory cytokine IL-10 actively down-regulates intestinal inflammatory process to prevent an excessive and prolonged inflammation. ${ }^{24)}$ In addition, it is reported that IL-10 deficiency could lead to a prolonged gastrointestinal dysmotility after surgery and abnormal high gene expression of pro-inflammatory cytokines in the animal model of POI. ${ }^{39)}$ In the present study, we found that POI rats showed significantly higher levels of pro-inflammatory cytokine TNF- $\alpha$, IL- $1 \beta$ and IL-6 in serum and the ileum tissue, while these elevated levels were significantly reduced by GRb1 in a dose-dependent manner, as well as their gene expression in the ileum tissue. In addition, although the level of anti-inflammatory cytokine IL-10 in serum and the ileum tissue was significantly increased in POI rats, GRb1 also continuously increased this elevated level in a dose-dependent manner, as well as this gene expression in the ileum tissue. These results suggested that GRb1 could reduce systemic and intestinal inflammation not only by decreasing the level of pro-inflammatory cytokine, but also increasing the level of anti-inflammatory cytokine in the animal model of POI.

In addition, MPO is an bioactive enzyme abundantly stored in azurophilic granules of neutrophils. It could be released into extracellular fluid in a state of inflammatory process. Therefore, the level of MPO activity has been commonly employed as a sensitive index to evaluate the extent of inflammatory response in various inflammatory disease. ${ }^{40)}$ In the present study, we found that POI rats showed significantly higher level of MPO activity in the ileum tissue, while this elevated level was significantly reduced by GRb1 in a dose-dependent manner. Combined with above cytokine results, we speculated that GRb1 may exhibit the effective anti-inflammatory activity by the inhibition of spleen tyrosine kinase, ${ }^{41)}$ the stimulation of parasympathetic pathways, ${ }^{21)}$ or the regulation of inducible nitric oxide synthase. ${ }^{42}$

In conclusion, our study demonstrates that GRb1 has a potent anti-inflammatory effect on POI and further contributes 
to the recovery of gastrointestinal motility. GRb1 may be a promising treatment for POI prophylaxis. However, GRb1 has multiple pharmacological effects, such as improvement of energy metabolism, oxidative stress and organ function., ${ }^{7,8}$ Therefore, a further study is required to determine the exact mechanism of GRbl's contribution to the recovery of gastrointestinal motility in POI.

Acknowledgments The authors would like to thank Prof. Qiurong Li, Research Institute of General Surgery at Jinling Hospital, for her excellent technical assistance. This study was supported by 12th five-year Major Program of Army Grants (AWS12J001); Jiangsu Province's Special Project of Science and Technology in Medicine (BL2012006).

\section{REFERENCES}

1) Sun J, Hu S, Song X. Adjuvant effects of protopanaxadiol and protopanaxatriol saponins from ginseng roots on the immune responses to ovalbumin in mice. Vaccine, 25, 1114-1120 (2007).

2) Xia X, Jiang B, Liu W, Wang P, Mou Y, Liu Y, Zhao Y, Bi X. Antitumor activity of three novel derivatives of ginsenoside on colorectal cancer cells. Steroids, 80, 24-29 (2014).

3) Sun C, Chen Y, Li X, Tai G, Fan Y, Zhou Y. Anti-hyperglycemic and anti-oxidative activities of ginseng polysaccharides in STZinduced diabetic mice. Food Func., 5, 845-848 (2014).

4) Chu S, Gu J, Feng L, Liu J, Zhang M, Jia X, Liu M, Yao D. Ginsenoside Rg5 improves cognitive dysfunction and beta-amyloid deposition in STZ-induced memory impaired rats via attenuating neuroinflammatory responses. Int. Immunopharmacol., 19, 317-326 (2014).

5) Lee HS, Kim MR, Park Y, Park HJ, Chang UJ, Kim SY, Suh HJ. Fermenting red ginseng enhances its safety and efficacy as a novel skin care anti-aging ingredient: in vitro and animal study. J. Med. Food, 15, 1015-1023 (2012).

6) Lee DC, Lau AS. Effects of Panax ginseng on tumor necrosis factor-alpha-mediated inflammation: a mini-review. Molecules, 16, 2802-2816 (2011).

7) Tan SJ, Li N, Zhou F, Dong QT, Zhang XD, Chen BC, Yu Z. Ginsenoside Rb1 improves energy metabolism in the skeletal muscle of an animal model of postoperative fatigue syndrome. J. Surg. Res., 191, 344-349 (2014).

8) Tan S, Zhou F, Li N, Dong Q, Zhang X, Ye X, Guo J, Chen B, Yu $\mathrm{Z}$. Anti-fatigue effect of ginsenoside $\mathrm{Rb} 1$ on postoperative fatigue syndrome induced by major small intestinal resection in rat. Biol. Pharm. Bull., 36, 1634-1639 (2013).

9) Zhu J, Jiang Y, Wu L, Lu T, Xu G, Liu X. Suppression of local inflammation contributes to the neuroprotective effect of ginsenoside Rb1 in rats with cerebral ischemia. Neuroscience, 202, 342-351 (2012).

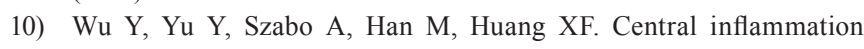
and leptin resistance are attenuated by ginsenoside Rb1 treatment in obese mice fed a high-fat diet. PLoS ONE, 9, e92618 (2014).

11) Joh EH, Lee IA, Jung IH, Kim DH. Ginsenoside Rb1 and its metabolite compound K inhibit IRAK-1 activation - the key step of inflammation. Biochem. Pharmacol., 82, 278-286 (2011).

12) Li QY, Chen L, Fu WH, Li ZD, Wang B, Shi XJ, Zhong MK. Ginsenoside Rb1 inhibits proliferation and inflammatory responses in rat aortic smooth muscle cells. J. Agric. Food Chem., 59, 6312-6318 (2011).

13) Li J, Zhong W, Wang W, Hu S, Yuan J, Zhang B, Hu T, Song G. Ginsenoside metabolite compound $\mathrm{K}$ promotes recovery of dextran sulfate sodium-induced colitis and inhibits inflammatory responses by suppressing NF-kappaB activation. PLoS ONE, 9, e87810 (2014). 14) Barletta JF, Senagore AJ. Reducing the burden of postoperative ileus: evaluating and implementing an evidence-based strategy. World J. Surg., 38, 1966-1977 (2014).

15) Boelens PG, Heesakkers FF, Luyer MD, van Barneveld KW, de Hingh IH, Nieuwenhuijzen GA, Roos AN, Rutten HJ. Reduction of postoperative ileus by early enteral nutrition in patients undergoing major rectal surgery: prospective, randomized, controlled trial. Ann. Surg., 259, 649-655 (2014).

16) Rychter J, Clave P. Intestinal inflammation in postoperative ileus: pathogenesis and therapeutic targets. Gut, 62, 1534-1535 (2013).

17) Vather R, Trivedi S, Bissett I. Defining postoperative ileus: results of a systematic review and global survey. J. Gastrointest. Surg., 17, 962-972 (2013).

18) Vather R, O'Grady G, Bissett IP, Dinning PG. Postoperative ileus: mechanisms and future directions for research. Clin. Exp. Pharmacol. Physiol., 41, 358-370 (2014).

19) Asgeirsson T, El-Badawi KI, Mahmood A, Barletta J, Luchtefeld M, Senagore AJ. Postoperative ileus: it costs more than you expect. $J$. Am. Coll. Surg., 210, 228-231 (2010).

20) van Bree SH, Nemethova A, Cailotto C, Gomez-Pinilla PJ, Matteoli G, Boeckxstaens GE. New therapeutic strategies for postoperative ileus. Nat. Rev. Gastroenterol. Hepatol., 9, 675-683 (2012).

21) Boeckxstaens GE, de Jonge WJ. Neuroimmune mechanisms in postoperative ileus. Gut, 58, 1300-1311 (2009).

22) Doorly MG, Senagore AJ. Pathogenesis and clinical and economic consequences of postoperative ileus. Surg. Clin. North Am., 92, 259-272, viii (2012).

23) Tan S, Yu W, Lin Z, Chen Q, Shi J, Dong Y, Duan K, Bai X, Xu L, Li J, Li N. Peritoneal air exposure elicits an intestinal inflammation resulting in postoperative ileus. Mediators Inflamm., 2014, 924296 (2014).

24) Wehner S, Vilz TO, Stoffels B, Kalff JC. Immune mediators of postoperative ileus. Langenbecks Arch. Surg., 397, 591-601 (2012).

25) Kalff JC, Carlos TM, Schraut WH, Billiar TR, Simmons RL, Bauer AJ. Surgically induced leukocytic infiltrates within the rat intestinal muscularis mediate postoperative ileus. Gastroenterology, 117, 378-387 (1999).

26) Kalff JC, Schraut WH, Simmons RL, Bauer AJ. Surgical manipulation of the gut elicits an intestinal muscularis inflammatory response resulting in postsurgical ileus. Ann. Surg., 228, 652-663 (1998).

27) Stoffels B, Hupa KJ, Snoek SA, van Bree S, Stein K, Schwandt T, Vilz TO, Lysson M, Veer CV, Kummer MP, Hornung V, Kalff JC, de Jonge WJ, Wehner S. Postoperative ileus involves interleukin-1 receptor signaling in enteric glia. Gastroenterology, 146, 176-187 (2014).

28) Endo M, Hori M, Ozaki H, Oikawa T, Hanawa T. Daikenchuto, a traditional Japanese herbal medicine, ameliorates postoperative ileus by anti-inflammatory action through nicotinic acetylcholine receptors. J. Gastroenterol., 49, 1026-1039 (2014).

29) Lee SH, Jung BH, Kim SY, Lee EH, Chung BC. The antistress effect of ginseng total saponin and ginsenoside $\mathrm{Rg} 3$ and $\mathrm{Rb} 1$ evaluated by brain polyamine level under immobilization stress. Pharmacol. Res., 54, 46-49 (2006).

30) Li YY, Cao MH, Goetz B, Chen CQ, Feng YJ, Chen CJ, Kasparek MS, Sibaev A, Storr M, Kreis ME. The dual effect of cannabinoid receptor-1 deficiency on the murine postoperative ileus. PLOS ONE, 8, e67427 (2013).

31) Bradford MM. A rapid and sensitive method for the quantitation of microgram quantities of protein utilizing the principle of proteindye binding. Anal. Biochem., 72, 248-254 (1976).

32) Shen J, Yu W, Chen Q, Shi J, Hu Y, Zhang J, Gao T, Xi F, He C, Gong J, Li N, Li J. Continuous renal replacement therapy (CRRT) attenuates myocardial inflammation and mitochondrial injury induced by venovenous extracorporeal membrane oxygenation (VV ECMO) in a healthy piglet model. Inflammation, 36, 1186-1193 (2013). 
33) Livak KJ, Schmittgen TD. Analysis of relative gene expression data using real-time quantitative PCR and the 2(-delta delta $\mathrm{C}(\mathrm{T})$ ) Method. Methods, 25, 402-408 (2001).

34) Ng SS, Leung WW, Mak TW, Hon SS, Li JC, Wong CY, Tsoi KK, Lee JF. Electroacupuncture reduces duration of postoperative ileus after laparoscopic surgery for colorectal cancer. Gastroenterology, 144, 307-313 (2013).

35) Choi HK, Lee YJ, Lee YH, Park JP, Min K, Park H. Inflammatory responses in the muscle coat of stomach and small bowel in the postoperative ileus model of guinea pig. Yonsei Med. J., 54, 1336-1341 (2013).

36) Shafiq N, Malhotra S, Pandhi P. Effect of cyclooxygenase inhibitors in postoperative ileus: an experimental study. Methods Find. Exp. Clin. Pharmacol., 24, 275-278 (2002).

37) Schmidt J, Stoffels B, Chanthaphavong RS, Buchholz BM, Nakao A, Bauer AJ. Differential molecular and cellular immune mechanisms of postoperative and LPS-induced ileus in mice and rats. Cytokine, 59, 49-58 (2012).

38) Wehner S, Schwarz NT, Hundsdoerfer R, Hierholzer C, Tweardy
DJ, Billiar TR, Bauer AJ, Kalff JC. Induction of IL-6 within the rodent intestinal muscularis after intestinal surgical stress. Surgery, 137, 436-446 (2005).

39) Stoffels B, Schmidt J, Nakao A, Nazir A, Chanthaphavong RS, Bauer AJ. Role of interleukin 10 in murine postoperative ileus. Gut, 58, 648-660 (2009).

40) Nussbaum C, Klinke A, Adam M, Baldus S, Sperandio M. Myeloperoxidase: a leukocyte-derived protagonist of inflammation and cardiovascular disease. Antioxid. Redox Signal., 18, 692-713 (2013).

41) van Bree SH, Gomez-Pinilla PJ, van de Bovenkamp FS, Di Giovangiulio M, Farro G, Nemethova A, Cailotto C, de Jonge WJ, Lee K, Ramirez-Molina C, Lugo D, Skynner MJ, Boeckxstaens GE, Matteoli G. Inhibition of spleen tyrosine kinase as treatment of postoperative ileus. Gut, 62, 1581-1590 (2013).

42) Türler A, Kalff JC, Moore BA, Hoffman RA, Billiar TR, Simmons RL, Bauer AJ. Leukocyte-derived inducible nitric oxide synthase mediates murine postoperative ileus. Ann. Surg., 244, 220-229 (2006). 